\title{
THE EFFECT OF SPATIAL COHERENCE OF SOURCES ON SYNTHETIC APERTURE MAPPING
}

\author{
DANIEL F. V. JAMES
}

The Institute of Optics, University of Rochester, Rochester, NY 14627, U.S.A.

The interferometric mapping of astronomical objects relies on the van-Cittert Zernike theorem, one of the major results of the theory of partially coherent light [see, Born and Wolf (1980), chapter 10]. This theorem states that the degree of spatial coherence of the field from a distant spatially incoherent source is proportional to the Fourier transform of the intensity distribution across the source. Measurement of the degree of spatial coherence, by, for example, measuring the visibility of interference fringes, allows the object to be mapped by making an inverse Fourier transform. (For a full description of this technique see Thompson, Moran and Swenson, 1986.)

In this paper I present a summary of the results an investigation into what happens when the distant source is not spatially coherent (James, 1990). Using a heuristic model of a spherically symmetric partially coherent source, an analytic expression for the error in the measurement of the effective radius, expressed as a function of coherence area, can be obtained.

The coherence function of a statistically stationary random scalar field is defined by

$$
\Gamma\left(\mathbf{r}_{1}, \mathbf{r}_{2}, \tau\right)=\left\langle\mathrm{V}\left(\mathbf{r}_{1}, t+\tau\right) V^{*}\left(\mathbf{r}_{2}, t\right)\right\rangle,
$$

where the angular brackets denote ensemble averages, and $V(r, t)$ is the complex analytic signal representation of the real valued field, for example, a Cartesian component of the electric vector [see Born and Wolf (1980) sec. 10.2]. We assume that $V(r, t)$ obeys an inhomogeneous wave equation,

$$
\nabla^{2} \mathrm{~V}(\mathbf{r}, \mathrm{t})-\frac{1}{\mathrm{c}^{2}} \frac{\partial^{2} \mathrm{~V}(\mathrm{r}, \mathrm{t})}{\partial \mathrm{t}^{2}}=-4 \pi \mathrm{Q}(\mathbf{r}, \mathrm{t}),
$$

where $c$ is the speed of light and $Q(r, t)$ is a random primary source distribution. It will be useful to employ the cross-spectral density function

$$
W\left(\mathbf{r}_{1}, \mathbf{r}_{2}, \omega\right)=\frac{1}{2 \pi} \int_{-\infty}^{\infty} \Gamma\left(\mathbf{r}_{1}, \mathbf{r}_{2}, \tau\right) \exp (i \omega \tau) d \tau .
$$

Coherence theory in the space-frequency representation, instead of the traditional space-time domain, has proved to be very useful for analysis of problems of this type. Two quantities 
of interest in the measurement of radiation fields can be obtained from the cross-spectral density function directly. The spectral intensity of the field at the point $\mathbf{r}$ is given by $S(r, \omega)=W(r, r, \omega)$ and the degree of spatial coherence at frequency $\omega$ is defined as [Mandel and Wolf (1976)]

$$
\mu\left(\mathbf{r}_{1}, \mathbf{r}_{2}, \omega\right)=\frac{W\left(\mathbf{r}_{1}, \mathbf{r}_{2}, \omega\right)}{\sqrt{W\left(\mathbf{r}_{1}, \mathbf{r}_{1}, \omega\right) W\left(\mathbf{r}_{2}, \mathbf{r}_{2}, \omega\right)}}
$$

The modulus of the degree of spatial coherence is equal to the visibility of fringes obtained in an interference experiment carried out with narrowband spectral filters centred at the frequency $\omega$ (Wolf, 1983).

It can be shown that the cross-spectral density of the far-zone field is given by the formula [see Carter and Wolf (1981a)]

$$
\begin{aligned}
& W^{(\infty)}\left(r_{1} s_{1}, r_{2} s_{2}, \omega\right)= \\
& \quad \frac{\exp \left[i k\left(r_{1}-r_{2}\right)\right]}{r_{1} r_{2}} \iint W_{Q}\left(r_{1}^{\prime}, r_{2}^{\prime}, \omega\right) \exp \left[-i k\left(s_{1} \cdot r^{\prime}{ }_{1}-s_{2} \cdot r^{\prime}{ }_{2}\right)\right] d^{3} r^{\prime}{ }_{1} d^{3} r_{2}^{\prime},
\end{aligned}
$$

where $k=\omega / c=2 \pi / \lambda, r_{1} s_{1}$ and $r_{2} s_{2}$ are the position vectors of two far zone points defined with respect to an origin in the vicinity of the source ( $s_{1}$ and $s_{2}$ being unit vectors) and $\mathrm{W}_{\mathrm{Q}}\left(\mathbf{r}^{\prime}{ }_{1}, \mathbf{r}^{\prime}{ }_{2}, \omega\right)$ is the cross-spectral density of the primary source distribution $\mathrm{Q}(\mathbf{r}, \mathfrak{t})$. The spatial integration is taken twice independently over the source domain.

We will now consider a Schell model source (Schell, 1967). Sources of this type have a degree of spatial coherence which is a function of the difference $r_{2}^{\prime}{ }_{2}^{-r^{\prime}}{ }_{1}$, only; the cross-spectral density is therefore of the form:

$$
\mathrm{W}_{\mathrm{Q}}\left(\mathbf{r}_{1}^{\prime}, \mathbf{r}_{2}^{\prime}, \omega\right)=\sqrt{\mathrm{S}_{\left.\mathrm{Q}^{(} \mathbf{r}_{1}^{\prime}, \omega\right) \mathrm{S}_{\mathrm{Q}}\left(\mathbf{r}_{2}^{\prime}, \omega\right)} \mu_{\mathrm{Q}_{2}}\left(\mathbf{r}_{2}^{\prime}-\mathbf{r}_{1}^{\prime}, \omega\right)},
$$

where $S_{Q}\left(r^{\prime}, \omega\right)$ is the spectral intensity of the source distribution and $\mu_{Q}\left(r^{\prime}{ }_{2}-r_{1}{ }_{1}, \omega\right)$ is its degree of spatial coherence at frequency $\omega$. We will choose for the spectral intensity a Gaussian function,

$$
S_{Q}(r, \omega)=I_{0}(\omega) \exp \left(-\frac{r^{2}}{2 a^{2}}\right)
$$

where $a$ is its effective radius of the source. We will model the degree of spatial coherence of the source also by a Gaussian function: 


$$
\mu_{\mathrm{Q}}\left(\mathbf{r}^{\prime}, \omega\right)=\exp \left(-\frac{\mathrm{r}^{2}}{2 \sigma^{2}}\right)
$$

where $\sigma$ is the effective coherence length at frequency $\omega$ of the fluctuating source distribution.

Substituting and performing the integration (for details, see Carter and Wolf, 1981b; James, 1990) we find that the degree of spatial coherence in the far zone is given by

$$
\mu^{(\infty)}\left(r_{1} s_{1}, r_{2} s_{2}, \omega\right)=\exp \left(-(2 \pi)^{2} \frac{a^{2} \delta^{2}}{\sigma^{2}} \frac{\left(u^{2}+v^{2}\right)}{2 r_{1}^{2}}\right) \exp (-i 2 \pi w) .
$$

where

$$
\frac{1}{\delta^{2}}=\frac{1}{4 \mathrm{a}^{2}}+\frac{1}{\sigma^{2}},
$$

and the baseline vector is given by $b=r_{1} s_{1}-r_{2} s_{2}=\lambda(u, v, w)$. Ignoring the geometric delay term as we are not interested in the position of the source but only in its dimensions and neglecting the effect of antenna response the visibility pattern measured in the $u, v$ plane is, therefore,

$$
\mathcal{V}(\mathrm{u}, \mathrm{v})=\exp \left(-(2 \pi)^{2} \frac{\mathrm{a}^{2} \delta^{2}}{\sigma^{2}} \frac{\left(\mathrm{u}^{2}+\mathrm{v}^{2}\right)}{2 \mathrm{r}_{1}^{2}}\right)
$$

The measured intensity distribution is (see Thompson et. al., Eq.(4.6) with antenna and geometric effects neglected)

$$
\begin{aligned}
\mathrm{B}(\xi, \eta) & \equiv \iint_{-\infty}^{8 q} \nu(\mathrm{u}, \mathrm{v}) \exp \mathrm{i} 2 \pi(\xi \mathrm{u}+\eta \mathrm{v}) \mathrm{du} \mathrm{dv} \\
& =\frac{1}{2 \pi}\left(\frac{\sigma \mathrm{r}_{1}}{\mathrm{a} \delta}\right)^{2} \exp \left[-\left(\frac{\sigma}{\mathrm{a} \delta}\right)^{2} \frac{\mathrm{r}_{1}^{2}\left(\xi^{2}+\eta^{2}\right)}{2}\right],
\end{aligned}
$$

where $\xi$ and $\eta$ are the angular coordinates of position on the celestial sphere. We see that the measured radius, $a_{\text {meas' }}$ ( $i$. e. the half width of the Gaussian function) is smaller than the actual radius of the source, a ; more precisely 


$$
\frac{a_{\text {meas }}}{a}=\frac{\delta}{\sigma}=\left(1+\frac{\sigma^{2}}{4 a^{2}}\right)^{-1 / 2}
$$

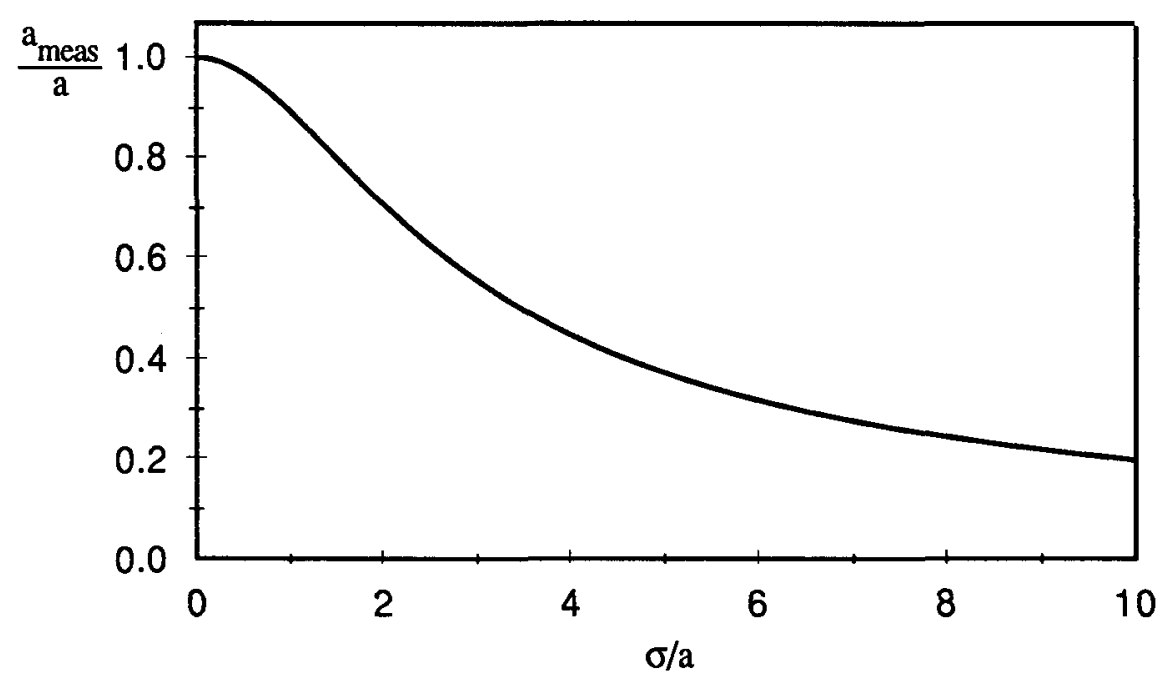

Fig.1 A graph of the ratio of measured radius to actual radius ( $\left.a_{\text {meas }} / \mathrm{a}\right)$ as a function of the ratio of the spatial coherence length of the source to the actual radius $(\sigma / \mathbf{a})$.

In conclusion, these calculations indicate that spatial coherence effects can effect the interferometric measurement of the radius of an object. It should be noted that this error will not occur in the images produced by conventional imaging telescopes.

\section{ACKNOWLEGDMENTS}

I would like to express my appreciation to Dan Watson for suggesting this research, and to Emil Wolf for helpful discussions and useful comments. This work was supported by the National Science Foundation and by the Army Research Office.

\section{REFERENCES}

Born, M. and Wolf, E. 1980, Principles of Optics, 6th ed.(Pergamon, Oxford).

Carter, W. H. and Wolf, E. 1981a, Optica Acta 28, 227 1981b, Optica Acta 28, 245.

James, D. F. V. 1990, Ap. J. 361, 650.

Mandel, L. and Wolf, E. 1976, J. Opt. Soc. Amer. 66, 529.

Schell, A. C. 1967, I. E. E. E. Trans. Antennas Propag. AP-15, 187.

Thompson, A. R., Moran, J. M. and Swenson, G. W. 1986, Interferometry and Synthesis in Radio Astronomy (Wiley, Chichester).

Wolf, E. 1983, Opt. Lett. 8, 250. 
Peter Dewdney: Can the presence of an intervening scattering medium provide spatial coherence, and if so, can these methods discussed in your talk be applied to calculate errors, etc.?

D. James: For non-forward scattering, weak scatterers are analogous to primary sources so that, if the incident light and the scattering medium are both highly correlated, the scattered light will be also. For multiple scattering (i.e. optically thick scattering media). I believe that the scattered light has correlation lengths which are small (of the order of a wavelength). 